\title{
Laser-Induced Fluorescence of Singly-Charged Xenon in a 6-kW Hall Thruster Plume
}

\author{
Wensheng Huang ${ }^{*}$, Bryan M. Reid ${ }^{\dagger}$, Timothy B. Smith ${ }^{\star}$, and Alec D. Gallimore ${ }^{\S}$ \\ Plasmadynamics and Electric Propulsion Laboratory, University of Michigan, Ann Arbor, MI 48109
}

We present laser-induced fluorescence velocimetry measurements of the $5 \mathrm{~d}[4]_{7 / 2} \rightarrow$ $6 \mathrm{p}[3]^{\circ}{ }_{5 / 2}$ transition for singly-charged xenon (Xe II) at $834.953 \mathrm{~nm}$ (vacuum) in the near-field plume of a 6-kW Hall thruster. Axial velocity profiles were obtained at six locations axially along the channel centerline and nine locations radially across the thruster channel at the exit plane. Discharge voltage was maintained at $300 \mathrm{~V}$, and the anode mass flow rate was set to 5,10 , and $20 \mathrm{mg} / \mathrm{s}$, corresponding to steady-state power levels of $1.5,3$, and $6 \mathrm{~kW}$. Since no published hyperfine structure constants were available for this transition, we used intermodulated optogalvanic spectroscopy to obtain an approximate lineshape for deconvolution of raw data. The acceleration zone was found to be on the order of few tens of millimeters in length. The loss voltage decreased from $45 \mathrm{~V}$ to $26 \mathrm{~V}$ as the mass flow rate was increased from 5 to $20 \mathrm{mg} / \mathrm{s}$. The bulk axial velocity at the exit plane peaked near the channel walls for all operating conditions. The axial velocity distribution along the thruster centerline exhibited a high-velocity tail, which may be due to charge-exchange effects. A lowvelocity tail was observed for near-wall velocity profiles.

\section{Nomenclature}

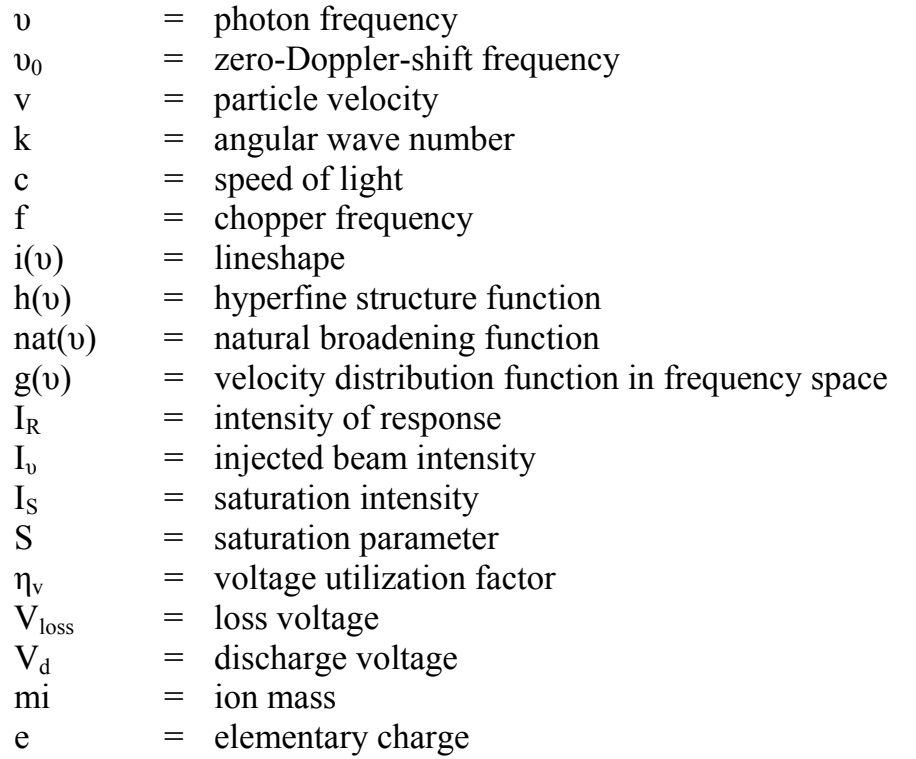

\section{Introduction}

THE performance envelope of the Hall thrusters is constantly being pushed through continual engineering refinements and testing. One of the areas currently being studied is the separation of the ionization and the

\footnotetext{
* Ph. D. Candidate, Aerospace Engineering, davhuang@umich.edu, Student Member, AIAA.

${ }^{\dagger} \mathrm{Ph}$. D. Candidate, Aerospace Engineering, reidb@umich.edu, Student Member, AIAA.

${ }^{\ddagger}$ Research Fellow and Lecturer, Aerospace Engineering, timsmith@umich.edu, Senior Member, AIAA.

$\S$ Arthur F. Thurnau Professor, Aerospace Engineering, rasta@umich.edu, Associate Fellow, AIAA.
} 
acceleration zones. The locations of these two zones are intricately tied in the Hall thruster. Ions born from collisions in the overlapping region between the ionization and acceleration zones do not execute the full potential drop provided by the discharge electrodes. This form of inefficiency as well as losses from ions being neutralized before reaching cathode potential are often characterized by a loss voltage term in the efficiency equation. ${ }^{1}$ The exact relationship between the ionization and the acceleration zones is difficult to characterize due to the limited number of truly non-intrusive near-field and internal studies that have been performed. Most of these studies involved some form of light-based diagnostic. ${ }^{2-4}$ Laser-induced fluorescence (LIF) is an optically based technique that requires no thruster modifications to obtain non-intrusive velocity distribution function (VDF) measurements.

LIF velocimetry measurements are taken along the discharge channel centerline of a $6-\mathrm{kW}$ thruster and the acceleration of ions near the exit is characterized. Characterization of the ionization zone, though possible with LIF, is not a part of the scope of this paper. Another phenomenon that is investigated in this paper is the influence of the magnetic field on ion acceleration at the exit plane of the 6-kW Hall thruster.

Due to the lack of published hyperfine structure data for the Xe II $5 \mathrm{~d}[4]_{7 / 2} \rightarrow 6 \mathrm{p}[3]_{5 / 2}^{0}$ transition, an intermodulated optogalvanic (IMOG) experiment is also performed to obtain a lineshape for deconvolution. Deconvolution is a mathematical tool that can be used to extract velocity distribution functions from raw LIF scans ${ }^{2}$. Although individual hyperfine splittings can not be resolved from the IMOG spectroscopy results, the results are sufficiently accurate to permit extraction of ion velocity distribution functions from LIF spectra.

\section{Theories}

Sections A-E lay out the theory underlying the extraction of VDFs from raw LIF spectra. Sections F and G briefly cover the phenomena of loss voltage and magnetic lensing in Hall thrusters, respectively. Note that for the remainder of the paper, all transition wavelengths are given in vacuum values and all intensities given in arbitrary units, a.u., unless otherwise specified. The use of arbitrary units is justified because the absolute magnitudes of intensities do not matter for the lineshapes in this study, only the relative magnitudes are important. Note that any discussion of temperature is avoided as the plasma under study is not expected to be in equilibrium given the discharge channel plasma conditions. Instead, full-width-at-half-maximum (FWHM) values are discussed.

\section{A. Principles of Laser-Induced Fluorescence Velocimetry}

LIF operates on the principle that when a particle (an atom or molecule) absorbs a photon, it will de-excite and emit another photon. Excited particles will de-excite by collision, spontaneous emission, or stimulated emission. Particles that spontaneously emit photons at the same energy as the absorbed photons are said to undergo resonant emission. Particles that emit photons at energies different from that of the absorbed photons are said to undergo nonresonant emission. This spontaneous emission, called fluorescence, radiates isotropically away from the particle.

To obtain the particle VDF via LIF, we take advantage of the Doppler shift.

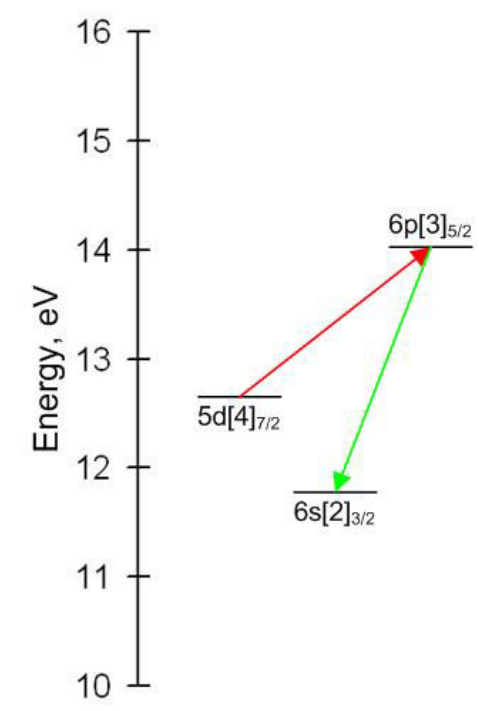

Figure 1. Transition diagram for Xe II LIF at $835 \mathrm{~nm}$.

For a particle travelling at non-relativistic speed, the shift in absorption frequency is proportional to the particle velocity component in the direction that the photon travels. The mathematical equation is given in Eq. (1)

$$
\frac{\Delta v}{v_{0}}=-\frac{\mathbf{v}}{\mathrm{c}} \cdot \frac{\mathbf{k}}{|\mathbf{k}|}
$$

where $v_{0}$ is the photon frequency, $\Delta v$ is the shift in photon frequency from the perspective of the particle, $\mathbf{v}$ is the particle velocity, $\mathrm{c}$ is the speed of light, and $\mathbf{k}$ is the photon wave vector. By varying the frequency of the injected photons and comparing the intensity of the collected fluorescence, we can obtain the particle VDF along the injected photon wave vector. In this experiment, light is injected at around $835 \mathrm{~nm}$ and non-resonant LIF is collected at $542.066 \mathrm{~nm}$. Figure 1 shows the transition diagram. Also, this experiment measures the VDF of the excited state $5 \mathrm{~d}[4]_{7 / 2}$, which is assumed to have the same VDF as the entire ion population. 


\section{B. Hyperfine Structure and Zeeman Splitting}

The FWHM of the average ion VDF in a Hall thruster typically varies from 800 to $2500 \mathrm{~m} / \mathrm{s}$, which for a nearinfrared transition corresponds to about 1000 to $3000 \mathrm{MHz}$. Unfortunately, xenon hyperfine structures typically have frequency shifts of $10 \mathrm{~s}$ to $1000 \mathrm{~s}$ of $\mathrm{MHz}$. These structures can add large distortions to the raw data. This effect shows up as multiple distinct spikes in a cold $(\mathrm{T}=0 \mathrm{~K})$ spectrum where there would normally only be one spike. This topic is dealt with in much greater details by Svanberg. ${ }^{5}$

Unfortunately, the hyperfine splittings for the Xe II $5 \mathrm{~d}[4]_{7 / 2} \rightarrow 6 \mathrm{p}[3]_{5 / 2}^{0}$ transition are large enough to broadened the raw lineshape but small enough such that no accurate determination of the lower state hyperfine structure constants have been made. Without experimentally-derived constants, we have no way to predict frequency shifts for this transition's 19 hyperfine split lines. If this hyperfine structure is not taken into account, the deconvolved width and shape of the velocity distribution functions will be inaccurate.

Furthermore, external electric and magnetic fields can alter the energies of otherwise degenerate states, creating a even greater number of hyperfine splittings. A more complete explanation and a set of modeling equations can be found in Ref. 5. For the purpose of this experiment, we will try to avoid this distortion as much as possible. These externally influenced splittings depends on the direction and polarity of the injected laser beam. The electric field present in a typical Hall thruster is several orders of magnitude smaller than what is necessary to cause a detectable frequency shift, called the Stark effect. ${ }^{6}$ The magnetic field effect for a weak $(\sim 100 \mathrm{G})$ field, called the Zeeman effect, can be categorized under $\sigma$ - and $\pi$-polarization. The beam is $\sigma$-polarized if the polarization of the beam is perpendicular to the external magnetic field $(\mathbf{E} \perp \mathbf{B})$. The beam is $\pi$-polarized if the polarization of the beam is parallel to the external magnetic field $(\mathbf{E} \| \mathbf{B})$. For the Xe II $5 \mathrm{~d}[4]_{7 / 2} \rightarrow 6 \mathrm{p}[3]^{\circ}{ }_{5 / 2}$ transition, $\pi$-polarized splittings are at least an order of magnitude lower than $\sigma$-polarized splittings. ${ }^{7}$ By injecting the laser beam with radial polarization to match the approximately radial magnetic field at the Hall thruster exit plane, we can obtain LIF scans with negligible Zeeman splitting. ${ }^{7}$ More in-depth descriptions of modeling the Zeeman effect on hyperfine splitting can be found in Ref. 8.

\section{Natural Broadening}

Natural (or lifetime) broadening is a result of nature following the Heisenberg uncertainty principle. Since energy and temporal measurements are complementary, we cannot know with absolute certainty the exact energy of a photon emitted from a particle whose decay time is uncertain. The result is that even if all other circumstances are equal, no two photons from the same de-excitation will be measured with exactly the same energy. This effect broadens an absorption line, modeled in frequency space as a Dirac delta function, into a Lorentzian function. ${ }^{5}$

\section{Principles of Deconvolution}

Deconvolution is a mathematical process that we can use to extract the particle VDF from a raw LIF spectrum. As described in the preceding sections, the raw data obtained from LIF is very messy but in a predictable fashion. Ignoring noise and other non-ideal effects, the lineshape in frequency space for a species with hyperfine splitting can be written as Eq. (2)

$$
\mathrm{i}(\mathrm{v})=\mathrm{h}(\mathrm{v}) \otimes \operatorname{nat}(\mathrm{v}) \otimes \mathrm{g}(\mathrm{v})
$$

where $h(v)$ is the hyperfine structure function, nat $(v)$ is the natural broadening function, and $g(v)$ is the VDF in frequency space. A raw LIF spectrum takes on the shape of $i(v)$, called the lineshape. Although the right hand side of the equation should include contributions by the laser beam profile, the linewidth of the diode laser used in this experiment is narrow enough for us to neglect the said profile. The $\otimes$ symbol represents the mathematical process called convolution, which is defined in Eq. (3)

$$
C(x)=\int_{-\infty}^{\infty} A(x-y) B(y) d y
$$

where the function $\mathrm{C}$ is the result of the convolution and the function $\mathrm{A}$ is said to be convolved with the function $\mathrm{B}$.

To obtain one of the original components of a convolution, we employ the deconvolution process, which is the inverse of the convolution process and is represented by the $\varnothing$ symbol. Equation (4) shows how the VDF, g(v), can 
be extracted from a raw LIF spectrum, i(v)

$$
\mathrm{g}(v)=\mathrm{i}(\mathrm{v}) \varnothing[\mathrm{h}(\mathrm{v}) \otimes \operatorname{nat}(v)]
$$

where $\mathrm{h}(v) \otimes$ nat $(v)$ is also called the kernel. In this case, the kernel require knowledge of the hyperfine constants and the lifetime of the Xe II $5 \mathrm{~d}[4]_{7 / 2} \rightarrow 6 \mathrm{p}[3]_{5 / 2}^{0}$ transition. Alternately, knowledge of the kernel alone (without knowledge of the individual hyperfine and natural broadening components) is sufficient to carry out deconvolution.

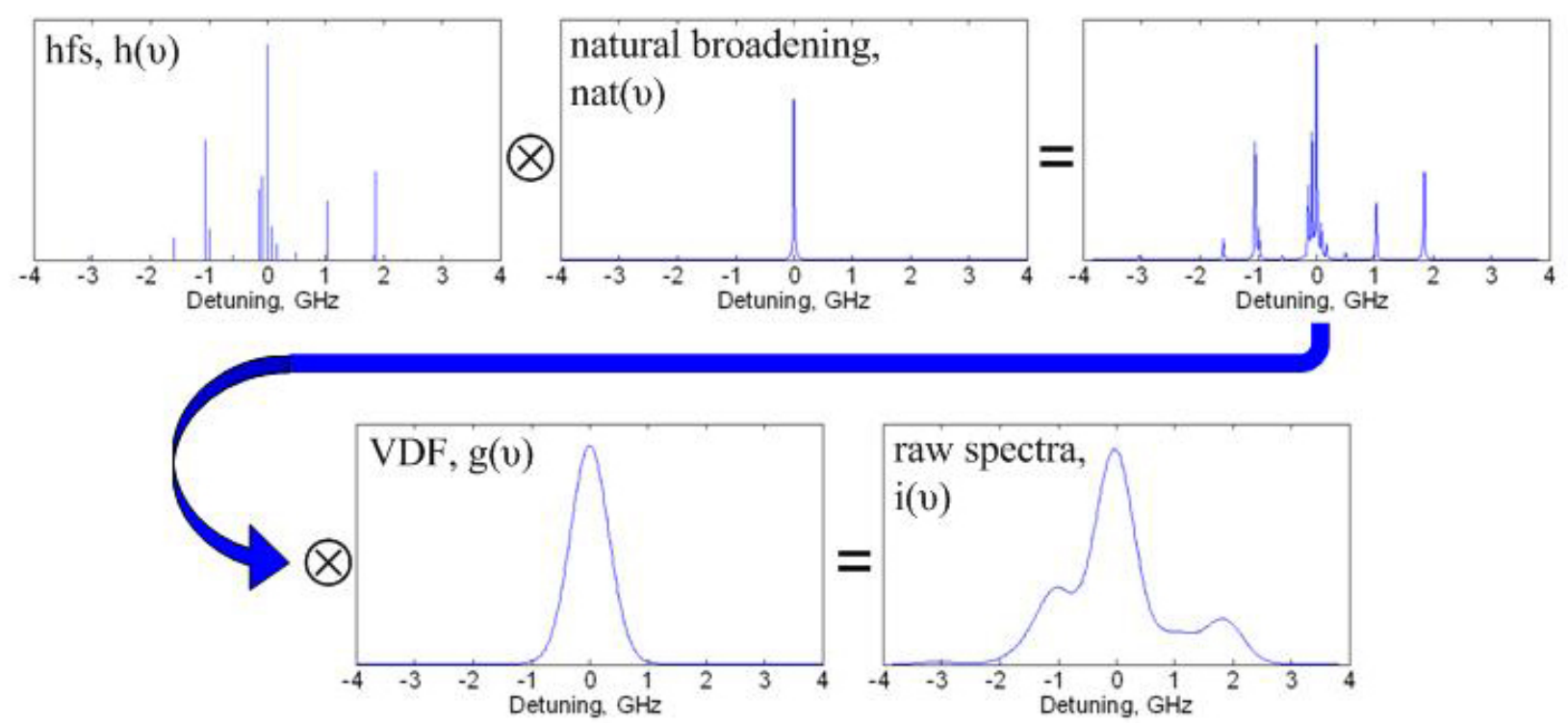

Figure 2. Graphical example of convolution for a typical xenon ion transition.

Graphically, convolution and deconvolution are both highly intuitive processes. Figure 2 shows an example of convolution represented by Eq. (2) for a typical xenon transition.

\section{E. Principles of Intermodulated Optogalvanic Spectroscopy}

Intermodulated optogalvanic spectroscopy (IMOG) is based on a non-ideal LIF effect called saturation. Physically, saturation happens when the beam intensity is high enough such that excited particles do not have enough time to de-excite before they are struck by another photon. At low intensity, the spontaneous emission rate is proportional to the input beam intensity. But as saturation sets in, the increase in spontaneous emission rate begins to decrease until the emission asymptotically reaches a maximum. At this point, the recorded emission signal becomes independent of the input beam intensity. For a two-level system, saturation can be modeled by Eq. $(5)^{5}$

$$
I_{R} \propto \frac{S(v)}{1+S(v)}, \text { where } S(v) \equiv \frac{I_{v}}{I_{S}(v)}
$$

where $I_{R}$ is the response (LIF or optogalvanic) of the sampled population, $S$ is called the saturation parameter, $I_{v}$ is the injected beam intensity, and $\mathrm{I}_{\mathrm{S}}$ is the saturation intensity. It can be shown that $\mathrm{I}_{\mathrm{S}}$ is the intensity of injected beam necessary to equally populate the upper and lower state of the transition in question. Note that $I_{S}(v)$ is frequency dependent and is inversely proportional to the magnitude of the lineshape $\mathrm{i}(v)$. For this reason, $\mathrm{S}(\mathrm{v})$ is proportional to $\mathrm{i}(v)$ and is higher at the peaks and lower at the troughs in the lineshape.

Normally, saturation is avoided because the tendency for peaks to be more saturated than troughs leads to an artificial broadening of the lineshape. However, saturation can be taken advantage of to produce a Doppler-free signal. From Eq. (2), we see that if the Doppler effect, $g(v)$, is removed, only the kernel remains. In this case, the kernel becomes a Doppler-free scan of the transition being examined. Depending on how well resolved the Dopplerfree scan is, hyperfine structure constants and natural linewidth can be approximated. 
To carry out IMOG, two laser beams of approximately equal intensity are sent from opposite directions to converge on the interrogation zone. Normally, one beam interacts with the population travelling one way while the other beam interacts with the population travelling in the opposite direction. When the frequencies of the beams match the zero-velocity frequency, both beams interact with the same population. If the interaction is linear, no change occurs in the response of the signal. However, if the intensities of the beams are high enough to produce strong saturation, the total response at the zero-velocity frequency will be different than the response at other frequencies. By modulating (chopping) the input beams at frequencies $f_{1}$ and $f_{2}$, respectively, a Doppler-free response is obtained at frequencies $\mathrm{f}_{1}+\mathrm{f}_{2}$ and $\mathrm{f}_{1}-\mathrm{f}_{2} \cdot{ }^{5}$ Note that the modulation frequency refers to the physical blocking and unblocking of the passage of light, and is not to be confused with the wave frequency of the passing light.

In practice, some Doppler broadening shows up in IMOG spectra due to velocity changing collisions that send particles going one way to go in the exact opposite direction. In addition, the two beams cannot be perfectly aligned. While these broadening effects are small, so is the Doppler-free signal. Defining the said broadening effects as noise, it can be shown with the saturation model equation, Eq. (5), that the signal-to-noise ratio (SNR) for an IMOG spectrum asymptotically reaches a maximum as the degree of saturation is increased. Realistically, the degree of saturation is dependent on the power of the laser and the ability to focus two beams onto the same interrogation volume. Note that the signal also theoretically maximizes for two beams having equal intensity and power given a total available power.

IMOG uses an optogalvanic cell as the response collection device. The cell is an enclosed space with the sample gas and two closely-spaced electrodes inside. When a particle is excited by the laser beam, it is more easily ionized by impacting electrons. This effect increases the measured current between the electrodes when a discharge is initiated. Since the cell contains a quiet, well-behaved plasma, intermodulated experiments performed in an optogalvanic cell can result in very high SNR.

\section{F. Hall Thruster Voltage Utilization}

The thrust efficiency of a Hall thruster can be written as a product of voltage, current, charge, and mass utilization factors. ${ }^{1}$ In particular, the voltage utilization factor can be readily inferred from axial LIF measurements. The definition of voltage utilization is given in Eq. (6)

$$
\eta_{\mathrm{v}} \equiv 1-\mathrm{V}_{\text {loss }} / \mathrm{V}_{\mathrm{d}}
$$

where $\mathrm{V}_{\text {loss }}$ is the loss voltage and $\mathrm{V}_{\mathrm{d}}$ is the discharge voltage. Assuming the sampled ions are travelling approximately axially and are all singly-charged, the difference between discharge voltage and loss voltage far away from the thruster can be calculated from bulk velocity measurement using Eq. (7)

$$
\mathrm{V}_{\mathrm{d}}-\mathrm{V}_{\text {loss }}=\frac{1}{2} \frac{\mathrm{m}_{\mathrm{i}} \mathrm{v}^{2}}{\mathrm{e}}
$$

where $\mathrm{m}_{\mathrm{i}}$ is the ion mass, $\mathrm{v}$ is the particle speed, and $\mathrm{e}$ is the elementary charge. Note that the left hand side of Eq. (7) is equal to the voltage drop experienced by the ions. The assumption of axial-only ion motion is justified because measurements used for this equation are taken along the centerline of the discharge channel where the divergence of the particles is small and causes negligible cosine error. The singly-charged assumption is justified because LIF spectra are species-specific. Combining axial LIF measurements with discharge voltage measurements, we can obtain the loss voltage and the voltage utilization.

\section{G. Magnetic Lensing Effect at the Exit Plane}

Advanced Hall thrusters incorporate a magnetic lens to focus plasma away from the walls and decrease beam divergence. ${ }^{9}$ However, because magnetic monopoles do not exist, the magnetic field gradient is directly tied to the magnetic field curvature. The implication is that for a finite geometry the magnetic field cannot decrease without curving. As a result, magnetic field lines will bulge out downstream of the exit plane. Since the magnetic field lines are generally representative of the equipotential lines, ${ }^{10}$ the plasma potential should peak near the discharge channel center. This effect has been previously measured with LIF for the P5 Hall thruster. ${ }^{11}$ 


\section{Experimental Apparatus and Procedures}

Subsections A-D describe the LIF experimental setup while subsection E describes the IMOG setup.

\section{A. Facility}

Experiments were performed in the Large Vacuum Test Facility (LVTF) of the Plasmadynamics and Electric Propulsion Laboratory (PEPL) at the University of Michigan. The LVTF is a $\varphi 6 \mathrm{~m} \times 9 \mathrm{~m}$ stainless steel-clad cylindrical chamber. Due to the size of the chamber, the thruster plume is able to expand un-impeded until termination at a beam dump $\sim 4 \mathrm{~m}$ downstream. Pumping is provided by seven cryopumps with a nominal xenon pumping speed of $240,000 \mathrm{l} / \mathrm{s}$. Facility pressure is monitored by two hot-cathode ionization gauges. The pressure values are given as the average of the two readings. The base pressure is approximately $2 \times 10^{-7}$ torr. The pressure during thruster operation at $10 \mathrm{mg} / \mathrm{s} \mathrm{xenon} \mathrm{mass} \mathrm{flow} \mathrm{rate} \mathrm{is} \mathrm{approximately} 8 \times 10^{-6}$ torr, corrected for xenon.

During the experiments, the $6-\mathrm{kW}$ Hall thruster was mounted on two cross-mounted translation stages. The stages provide $1 \mathrm{~m}$ of axial travel and $2 \mathrm{~m}$ of radial travel accurate to within $0.01 \mathrm{~mm}$. Laser injection and LIF collection optics were fixed from the ceiling of the chamber while the thruster was moved.

Research-grade xenon propellant $(99.999 \%$ pure) was supplied to the thruster by commercially available flow meters and controllers, having an accuracy of $\pm 1 \%$ of full scale. Calibration of the flow system was done by the constant volume method taking into account the effects of compressibility.

\section{B. Thruster}

The tested thruster is a 6-kW laboratory Hall thruster. Its nominal operating condition is at a discharge voltage of $300 \mathrm{~V}$ and an anode mass flow rate of $20 \mathrm{mg} / \mathrm{s}$. The thruster has inner, outer, and trim magnet coils but the trim coil was not used during this test. Neutralization was provided by a center-mounted $\mathrm{LaB}_{6}$ cathode. The magnet settings were chosen such that thrust efficiency was maximized as determined with an inverted pendulum thrust stand. More information on this thruster can be found in Ref. 12-14.

\section{Laser and Optics}

The laser system used in this experiment is a TUI Optics TA-100/830 tapered-amplifier diode laser. This system has a nominal linewidth of $\sim 5 \mathrm{MHz}$, a tuning range of $820 \mathrm{~nm}$ to $836 \mathrm{~nm}$, and a mode-hop-free range of $\sim 12 \mathrm{GHz}$.

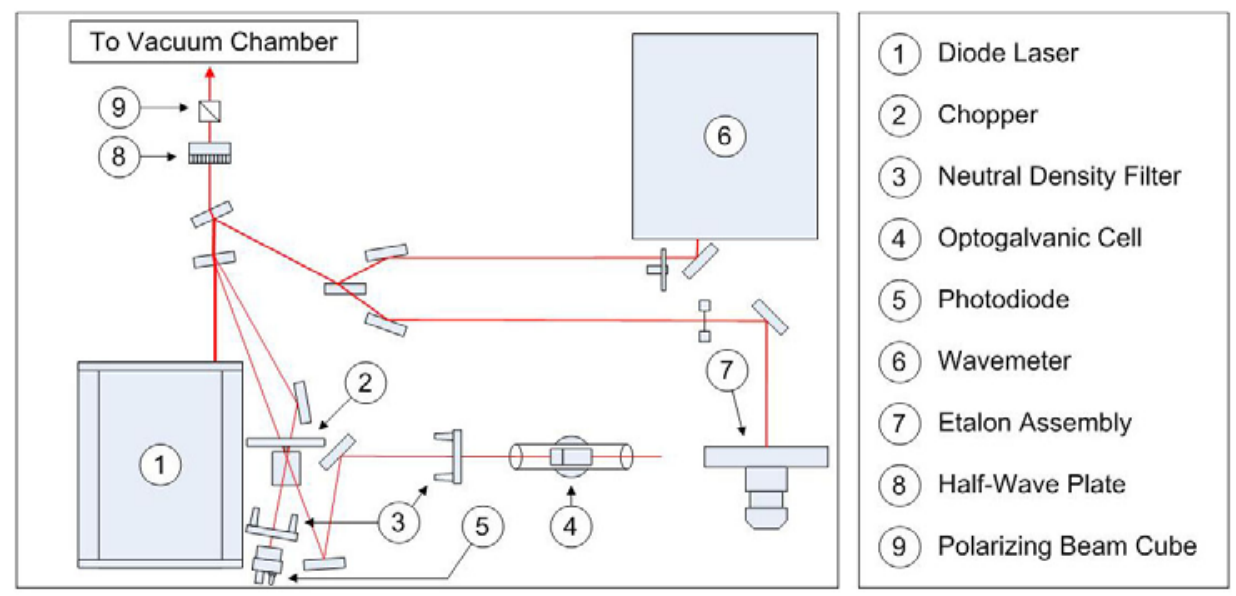

Figure 3. Air-side laser and optics setup for LIF experiment. Maximum output power is in excess of $500 \mathrm{~mW}$. Off-peak efficiency drops and Faraday isolation of the tapered amplifier reduced the available output power to $\sim 250 \mathrm{~mW}$ at 834.953 nm (vacuum).

The master oscillator for this system is an external cavity diode laser (ECDL). Coarse wavelength control is provided by Peltier cooling of the ECDL and micrometer adjustment of the ECDL feedback grating angle. Fine wavelength control is provided by a piezoelectric transducer (PZT) attached to the ECDL feedback grating. Feedback circuitry in the ECDL power supply decreases the master oscillator diode current during a PZT sweep to maximize the mode-hop-free range. Steering mirrors inside the TA-100 enclosure couple the ECDL output into the tapered amplifier diode, which boosts the ECDL output power by a factor of $\sim 50$ while maintaining the master oscillator wavelength and linewidth.

Figure 3 shows the schematic for the air-side laser and optics setup. The output of TA-100 first strikes an uncoated glass window, at which point two beams on the order of $\sim 5 \mathrm{~mW}$ are split off (one off of each interface of the window). These two beams will be referred to as beams A and B. The main beam then continues off to the first 
beam splitter, which reflects $\sim 40 \%$ of the power to a second beam splitter. The second beam splitter splits off two beams of about $\sim 50 \mathrm{~mW}$ each, which will be referred to as beams $\mathrm{C}$ and $\mathrm{D}$. The remaining laser power $(\sim 120 \mathrm{~mW})$ is refer to as beam $\mathrm{E}$.

Beam A goes through, in order, one SR540 chopper (at $\sim 1.6 \mathrm{kHz}$ ), one neutral density filter (NDF), and into one Hamamatsu optogalvanic cell. Originally, the cell was to be used as a stationary reference. However, due to the limited mode-hop-free range of the laser, the reference line was found to lie outside of a single LIF scan. Instead, we use a wavemeter to fix the reference frequency at the cost of increased absolute frequency uncertainty.

Beam B goes through the same chopper as beam A, one neutral density filter, and into a Thorlabs DET110 photodiode. An SR810 lock-in amplifier extracts the photodiode signal. Since the photodiode has a known efficiency and is connected to a preamplifier of known characteristics, this signal can track the beam power. The intensity recorded by this photodiode is used to correct for laser intensity variations with frequency as well as to carry out saturation studies.

Beam $\mathrm{C}$ is sent through one neutral density filter and into the Burleigh WA-1000 wavemeter. The wavemeter has a 1-pm resolution and provides coarse wavelength markers.

Beam D is sent through an iris and into the Burleigh SA-91 etalon assembly. The etalon assembly has a free spectral range of $2 \mathrm{GHz}$ and a finesse of 256 . Reference markers provided by the etalon can reduce the relative frequency uncertainty to the value of the scanning resolution, which is $\sim 30 \mathrm{MHz}$ for both LIF and IMOG experiments. This value is $0.25 \%$ of the full mode-hop-free range of the laser.

Beam $\mathrm{E}$ is sent through one half-wave plate, one polarizing cube, one chopper $(\sim 1.7 \mathrm{kHz})$, a sapphire window port, and into the vacuum-side periscope, mounted with its input window slightly above the middle of the chamber.

\section{Periscope and Collection Optics}

Figure 4 shows the beam-handling and collection optics setup used for the LIF experiment inside the LVTF. The beam that came through the sapphire window (which by this point is about $\sim 50 \mathrm{~mW}$ ) is redirected $1.4 \mathrm{~m}$ downstream of the thruster (parallel to the thruster axis), turned around, and sent axially into the thruster. Two lenses focus the beam waist down to $\sim 1 \mathrm{~mm}$ in diameter.

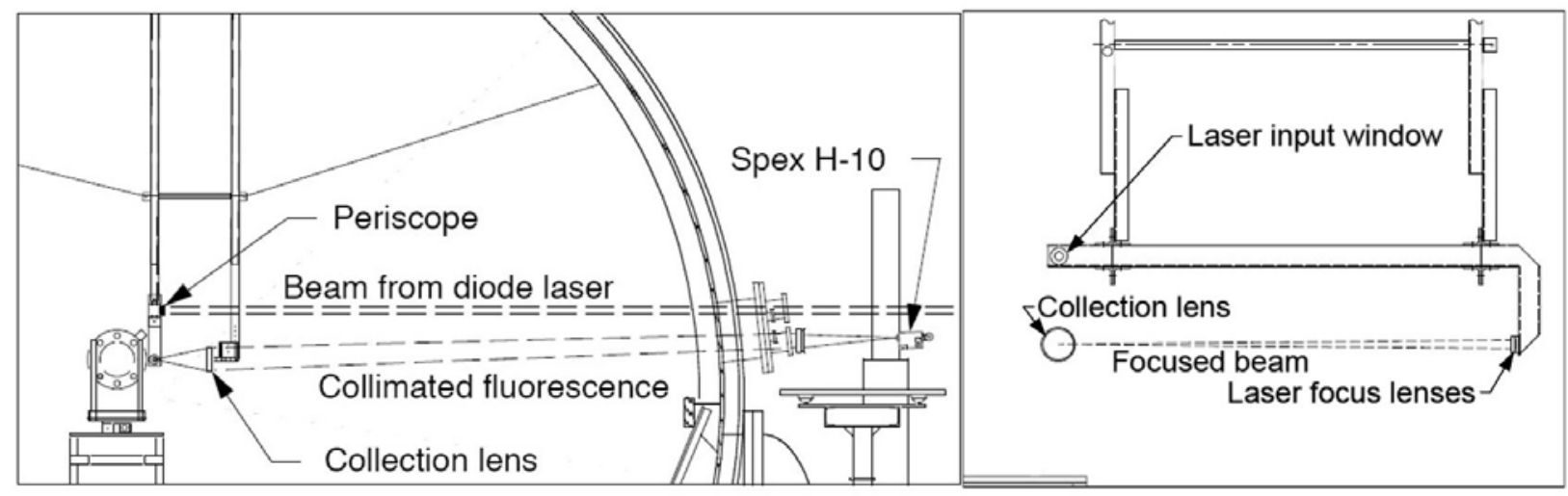

(a) Looking upstream (north),

(b) Looking west.

Figure 4. Vacuum side periscope and optics setup for LIF experiment.

Laser-induced fluorescence is collected by a $\varphi 100 \mathrm{~mm}, \mathrm{f} / 2.5$ lens. The collimate LIF is directed through another sapphire window and into a focusing lens, which focuses the fluorescence signal into a Jobin-Yvon H-10 monochromator. The monochromator input and exit slits were $0.5 \mathrm{~mm}$ wide and about $1 \mathrm{~cm}$ high. The combination of the periscope and monochromator slits limits the interrogation zone to a $\varphi 1 \mathrm{~mm} \times 1 \mathrm{~mm}$ cylinder oriented parallel to the thruster axis. An SR850 lock-in amplifier was used to extract the LIF signal from a Hamamatsu R928 photomultiplier coupled to the $\mathrm{H}-10$ exit.

\section{E. IMOG Setup and Optogalvanic Cell}

Figure 5 shows the laser and optics setup for the IMOG experiment. As with the LIF setup, two relatively weak beams are split off into the etalon assembly and the wavemeter for reference information. The remaining beam is split into pump beam A and pump beam B. 


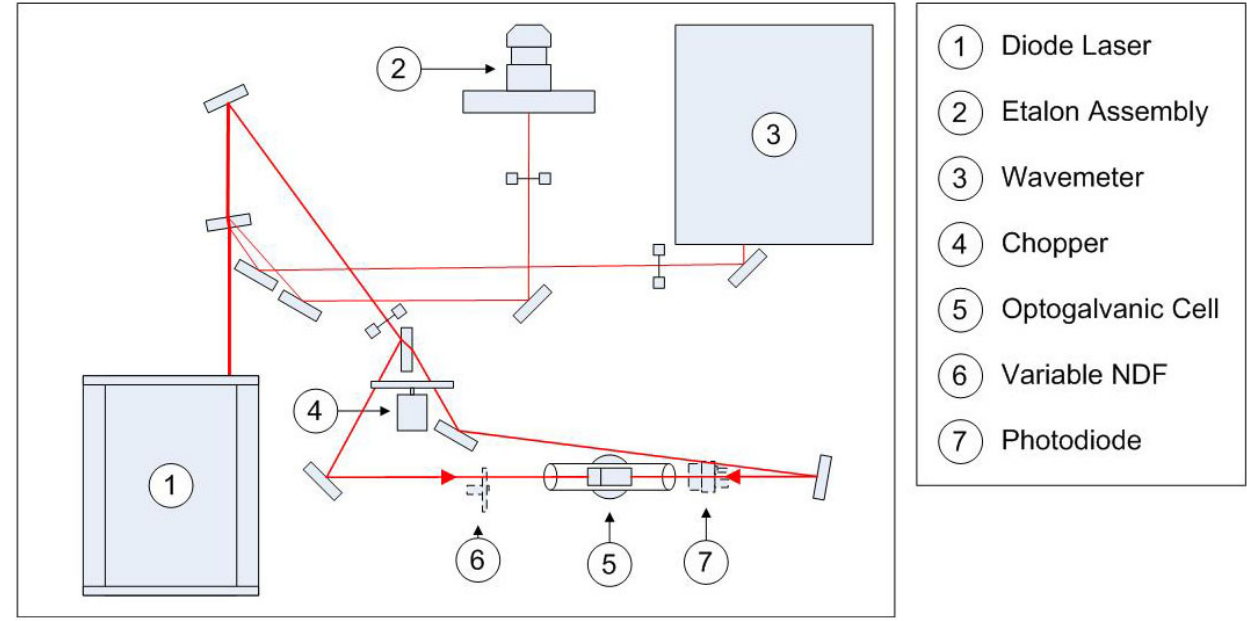

Figure 5. Laser and optics setup for the IMOG experiment.

Pump beam A, chopped at $1160 \mathrm{~Hz}$, is at $\sim 90 \mathrm{~mW}$ after the beam splitter. During saturation study, an additional variable NDF filter and a photodiode are added while pump beam B is blocked.

Pump beam B, chopped at $970 \mathrm{~Hz}$, is at $\sim 80 \mathrm{~mW}$ after the beam splitter. The IMOG signal is collected at the sum frequency of $2130 \mathrm{~Hz}$. The two beams are directed in the opposite directions into the optogalvanic cell. The beam axes of the two beams are within $0.1^{\circ}$ of each other.

The optogalvanic cell used in this study is a Hamamatsu L2783-42 XeNe-Mo galvatron. The core of the galvatron is a pair of $\varphi 6.25 \mathrm{~mm}$ cylindrical Mo electrodes centered in a $\varphi 25 \mathrm{~mm}$ x $120 \mathrm{~mm}$ glass cylinder. The cylinder is filled with approximately 3 torr of xenon and 4 torr of neon. The ends of the cylinder are angled approximately 10 degrees from being perpendicular to the electrode axis to eliminate retro-reflection. This design permits unobstructed passage of a laser beam along the axis shared by the cylinder and electrodes.

The galvatron is operated at $280 \mathrm{~V}$ to maintain a warm, dense, and stationary plasma. A ballast resistor prevents runaway current growth after the discharge is struck, and provides a simple method of measuring the discharge current. The voltage drop across the ballast resistor is connected to the SR-810 lock-in amplifier through an RC filter. The filter passes the AC component of the signal, while isolating the lock-in from the high DC component.

\section{Intermodulated Optogalvanic Spectroscopy Results and Validation}

Figure 6 shows the saturation study plot for the IMOG experiment. The line fitted to the saturation study is based on Eq. (5). The population is well saturated and the operating intensity is slightly greater than the saturation intensity, Is. Figure 7 shows the result of a single IMOG scan obtained with a lock-in time constant of $300 \mathrm{~ms}$.

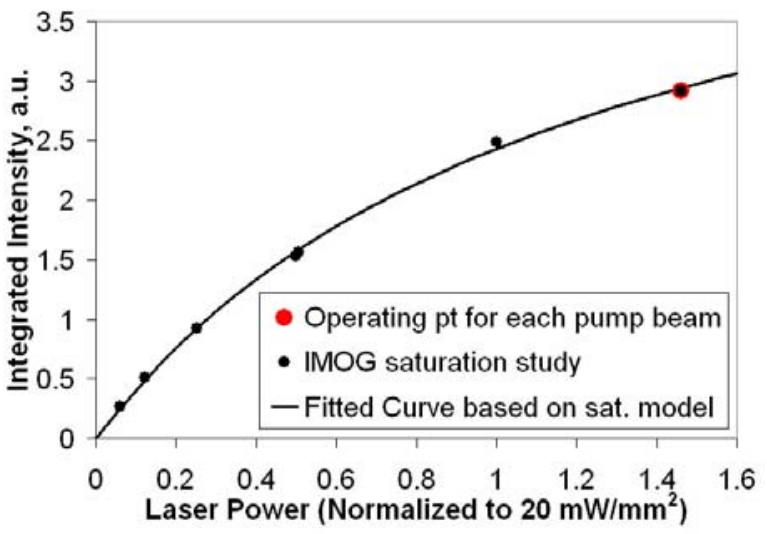

Figure 6. IMOG saturation study.

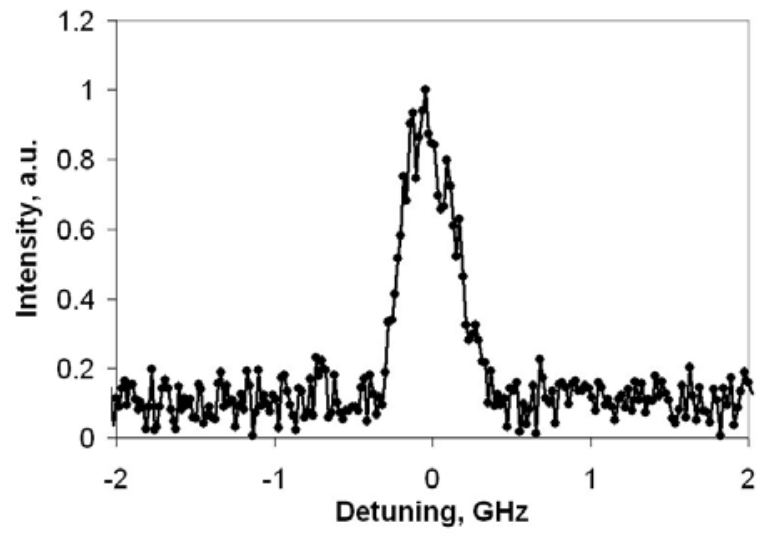

Figure 7. Sample IMOG scan.

Figure 8 shows the combined average of seven scans (solid line). Averaging was done by taking the centroid of each scan as a common reference point. Seven-point Savitsky-Golay smoothing ${ }^{15}$ was used to remove some of the noise. This smoothing scheme is equivalent to fitting a cubic equation onto seven points at a time and finding the value at the center. Due to the proximity of the Xe II $834.953 \mathrm{~nm}$ line to the Xe I $834.974 \mathrm{~nm}$ line, scans of the ion line contain spill-over signal from the neutral line. This spill-over is removed by assuming a linear trend on the spill- 


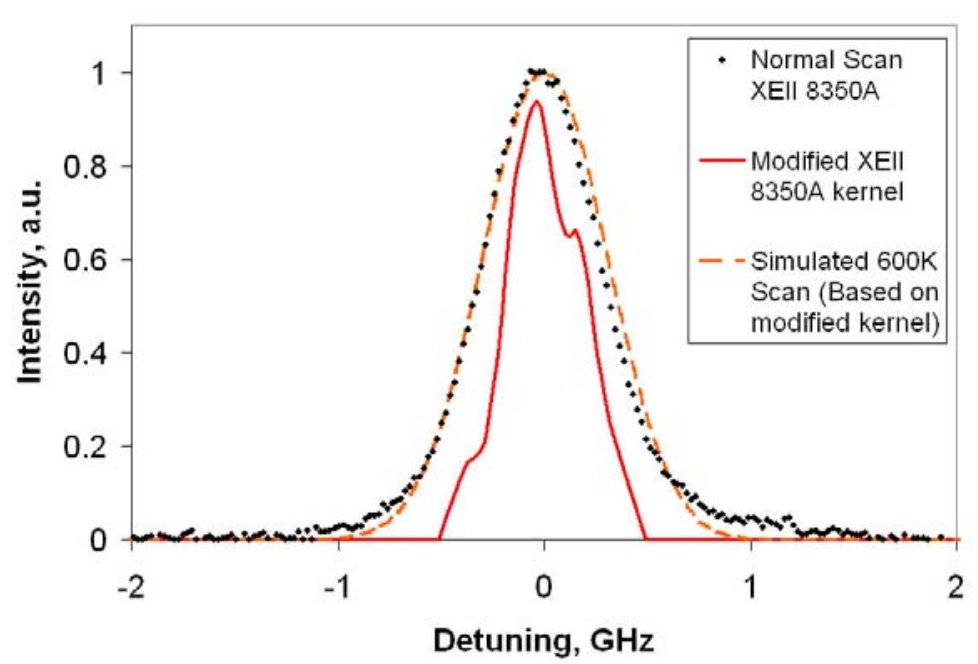

Figure 8. Modified kernel and normal scan for Xe II 834.953 nm (vacuum) transition.

over signal and subtracted from the data. Also shown in Fig. 8 in dots is a normal optogalvanic scan with an ion temperature of $600 \mathrm{~K}$ for comparison. A simulated $600-\mathrm{K}$ spectrum based on the modified kernel is also shown in dashed line.

From Fig. 7 and 8, we see that there is a large amount of Doppler-broadened noise mixed in with the Doppler-free signal. Sources of noise include velocity changing collisions and the slight misalignment of the laser.

Nevertheless, we have obtained several important pieces of information about the Xe II $5 \mathrm{~d}[4]_{7 / 2} \rightarrow 6 \mathrm{p}[3]_{5 / 2}^{0}$ transition. First, since the FWHM of the kernel is $440 \mathrm{MHz}$, we know that the majority of the 19 hyperfine split lines are within $\sim 500 \mathrm{MHz}$ of each other.

Second, there is a small plateau on the right side of the normal scan that does not show up on the IMOG scan. This plateau is most likely due to one or two low-intensity hyperfine lines, which tend not to show up in IMOG results because the lines are not well saturated. Lastly, from previous experiments ${ }^{7}$ with this optogalvanic cell, we have an estimated ion plasma temperature of $\sim 600 \mathrm{~K}$ at the voltage setting used. By simulating the Doppler broadening at this temperature, we see that the kernel does a fairly good job of simulating the true hyperfine structure of this transition despite the noise. Most likely, the hyperfine split lines are so close to each other that Doppler broadening at typical plasma temperatures smears out any hyperfine structure.

As a further study, we carried out deconvolution while ignoring hyperfine constants and natural broadening. Table 1 shows the results of this study. Gaussian smoothing filters of equal FWHM were applied to all deconvolution operations. As the table shows, the hyperfine splittings were so close together that not taking them into account produces only $\sim 2 \%$ broadening of the ion VDF in a typical Hall thruster plume. The table also shows that this broadening increases as the ions accelerate and, due to kinematic compression ${ }^{16}$, the ion VDF became narrower. On the other hand, neglecting the hyperfine structure will introduce an unknown amount of distortion to the resulting VDF. Thus, we would still have to take hyperfine structures into account in situations where the shape of the VDF matters, such as charge-exchange studies, and for making very-high-resolution VDF measurements.

Table 1.Comparison of results while taking and not taking into account hyperfine structure and natural broadening for Xe II $834.953 \mathrm{~nm}$ transition

\begin{tabular}{|c|c|c|c|}
\hline \multirow[b]{2}{*}{ Scan Conditions } & \multicolumn{2}{|c|}{ FWHM of velocity in results, $\mathbf{m} / \mathbf{s}$} & \multirow[b]{2}{*}{$\begin{array}{l}\text { Difference in } \\
\text { Percentage }\end{array}$} \\
\hline & $\begin{array}{l}\text { Using IMOG } \\
\text { kernel }\end{array}$ & $\begin{array}{l}\text { Ignore hfs and } \\
\text { natural broadening }\end{array}$ & \\
\hline $20 \mathrm{mg} / \mathrm{s}$, cent & 2747 & 2801 & $2 \%$ \\
\hline $20 \mathrm{mg} / \mathrm{s}$, centerline, $100 \mathrm{~mm}$ downstream & 2191 & 2245 & $2.5 \%$ \\
\hline
\end{tabular}

As will be seen later, the spectra collected for the LIF experiment have FWHM of around 1000 to $3000 \mathrm{MHz}$. We assume that the Doppler broadening noise in the kernel has a maximum FWHM of $440 \mathrm{MHz}$. Since Doppler broadening combines as the square root of the sum of squares of individual broadening effects, the contribution from the Doppler broadening noise amounts to small errors in the overall shape and FWHM of the VDF. This error is estimated to be $<1 \%$ for a scan with $3000 \mathrm{MHz}$ FWHM and $<10 \%$ for a scan with $1000 \mathrm{MHz}$ FWHM. At this point, the kernel was deemed acceptable for deconvolution purposes and refinement of the kernel to extract hyperfine constants was left for future work. 


\section{Laser-Induced Fluorescence Results}

LIF data were collected for three anode mass flow rates, 5,10 , and $20 \mathrm{mg} / \mathrm{s}$, with the discharge voltage held constant at $300 \mathrm{~V}$. Cathode mass flow rate was set to $7 \%$ of anode mass flow rate. The interrogation zone was moved radially across the channel at the exit plane as well as downstream along the channel centerline. In an attempt to cut down on thermal drift, the lock-in time constant (and therefore the total scan time) was kept as low as possible while still extracting meaningful data. The majority of the scans were done with a 300-ms time constant. A few scans at more than $100 \mathrm{~mm}$ downstream from the exit plane were done with a 1-s time constant. All scans have a frequency resolution of $30 \mathrm{MHz}$. Due to the presence of noise, an inverse Gaussian filter was applied to each scan before deconvolution. The FWHM of the filter varies from $560 \mathrm{MHz}$ to $770 \mathrm{MHz}$ depending on the SNR of the scan. All deconvolutions used the kernel shown in Fig. 8. Bulk velocities were accurate to within $3 \%$ and the corresponding potentials were accurate to within $6 \%$.

Figure 9 shows the results of a saturation study for the LIF experiment. No saturation was observed over the range of laser powers used in the LIF experiment.

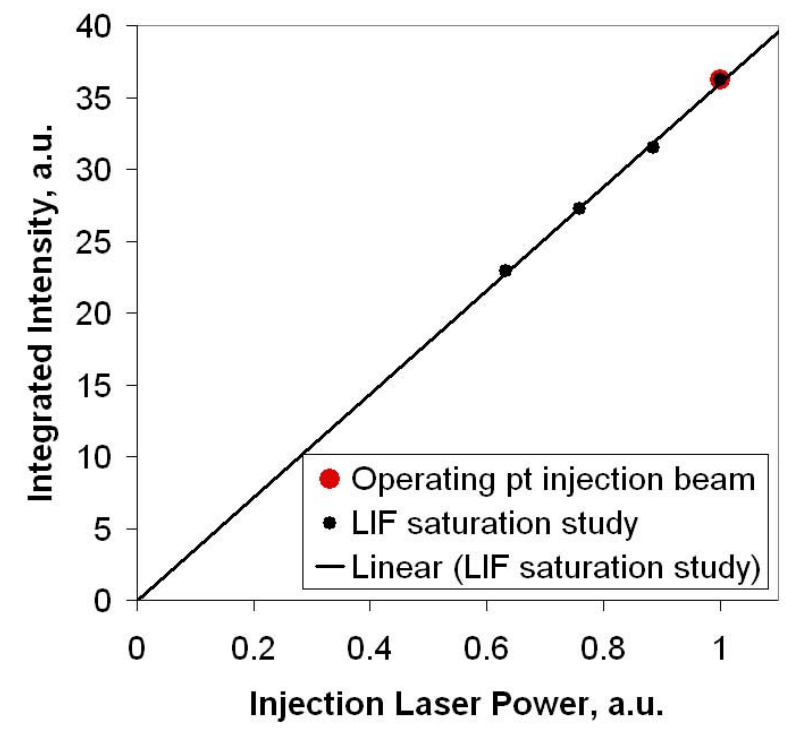

Figure 9. LIF saturation study plot.

\section{A. Bulk Velocity Results Axially along Channel Centerline}

Figure 10 shows the bulk velocity along the channel centerline for all three operating conditions. Bulk velocity was calculated as the centroid of the velocity distribution function. Since the bulk velocity was calculated from all data points in a scan, its uncertainty is only limited by the resolution of the wavemeter. Uncertainty in axial location was $<1 \mathrm{~mm}$. The data indicate that the loss voltage is approximately the same for the 10 and $20 \mathrm{mg} / \mathrm{s}$ cases, but is notably higher for the $5 \mathrm{mg} / \mathrm{s}$ case. The 10 and $20 \mathrm{mg} / \mathrm{s}$ cases exhibit similar acceleration profiles, where particles reach terminal velocities within 20-30 $\mathrm{mm}$ downstream of the exit plane. The data for the $5 \mathrm{mg} / \mathrm{s}$ case indicate a lengthier acceleration process that extends out to 50-100 mm downstream.

Figure 11 shows the plasma potential calculated from the bulk velocity based on the LIF results. Internal measurements recorded by Reid with a high-speed positioning table and emissive probe are also shown for comparison. ${ }^{17}$

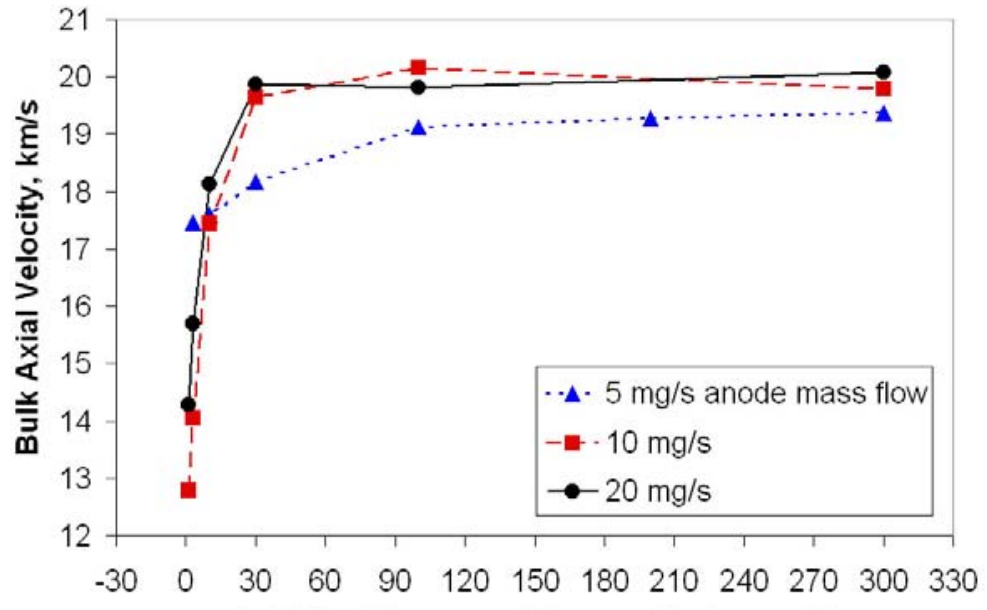

Axial Position, $\mathrm{mm}$ (Thrust exit plane at 0 )

Figure 10. Bulk axial velocity along the thruster channel centerline at various mass flow rates and $300 \mathrm{~V}$ discharge. 


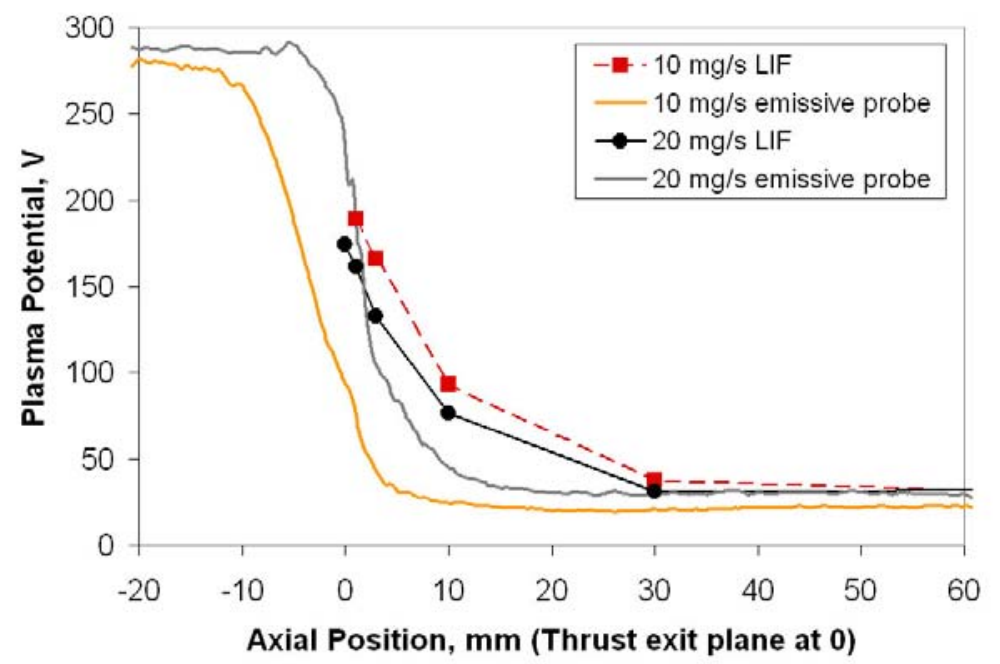

Figure 11. Plasma potential calculated from LIF data compared to emissive HARP data.
Table 2. Loss voltage and voltage utilization at various anode mass flow rates.

\begin{tabular}{lll}
\hline \hline $\begin{array}{l}\text { Anode } \\
\text { mass flow }\end{array}$ & $\begin{array}{l}\text { Loss } \\
\text { Voltage, }\end{array}$ & $\begin{array}{l}\text { Voltage } \\
\text { Utilization }\end{array}$ \\
rate, mg/s & V & \\
\hline 5 & $45 \pm 3$ & 0.85 \\
10 & $33 \pm 2$ & 0.89 \\
20 & $26 \pm 2$ & 0.91 \\
\hline \hline
\end{tabular}

The shapes of the data are similar for both measurement techniques, but the location of the acceleration zone differs by up to $7 \mathrm{~mm}$. This discrepancy was not expected and requires further investigations. The potential values downstream of the acceleration zone agree to within $10 \mathrm{~V}$ for all corresponding measurements.

Table 2 shows the loss voltage and voltage utilization as computed from LIF data at $300 \mathrm{~mm}$ downstream of the exit plane. Voltage utilization appears to decrease with anode mass flow rate away from the nominal operating condition of the 6-kW Hall thruster.

\section{B. Bulk Axial Velocity Results Radially across Thruster Exit Plane}

Figure 12 shows radial variations in the axial bulk velocity at the thruster exit plane for operation at 5,10 , and $20 \mathrm{mg} / \mathrm{s}$ anode flow rates. A few data points near the edge are not plotted due to low SNR. The bulk velocity dips near the center of the channel for all operating conditions. As discussed in section II.G, plasma potential is expected to be the highest at the center in a radial sweep at the exit plane. The observed trend is consistent with our expectations because plasma with higher potential has undergone less acceleration. The potential difference between centerline plasma and edge plasma is approximately $15 \mathrm{~V}$ for all three flow rates.

We believe the difference in bulk axial velocity at different mass flow rates was primarily due to slight variances in the plasma location from test to test. The exact relationship between the mass flow rate and the location of the main bulk of the plasma was not clear in this experiment.

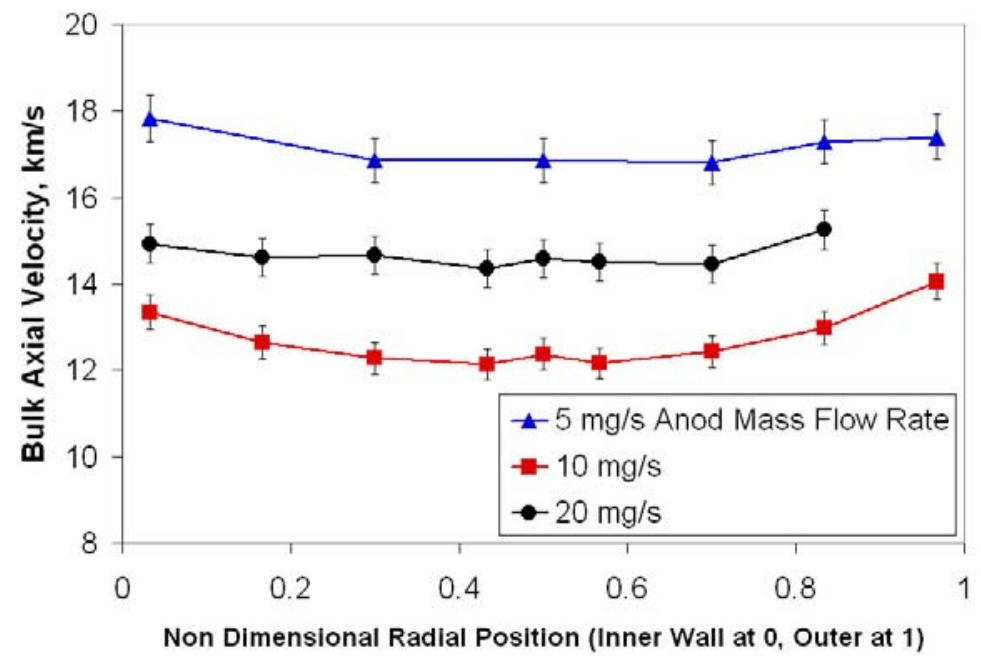

Figure 12. Bulk axial velocity radially across the discharge channel at thruster exit plane for various flow rates and $300 \mathrm{~V}$ discharge.

\section{Velocity Distribution Functions}

Figure 13 shows how the velocity distribution function varies as a function of downstream position at the thruster channel centerline for a $300 \mathrm{~V}, 20 \mathrm{mg} / \mathrm{s}$ discharge. Due to the limited mode-hop-free range of the laser, some scans are cut off at one end. Nevertheless, almost all of the distributions are positive-skewed, indicating the presence high-energy tails. The exceptions are the $10-\mathrm{mm}$ scan, which is cut off toward the high-energy end, and the $300-\mathrm{mm}$ scan, which is noisy enough that the presence of tails is not obvious. 


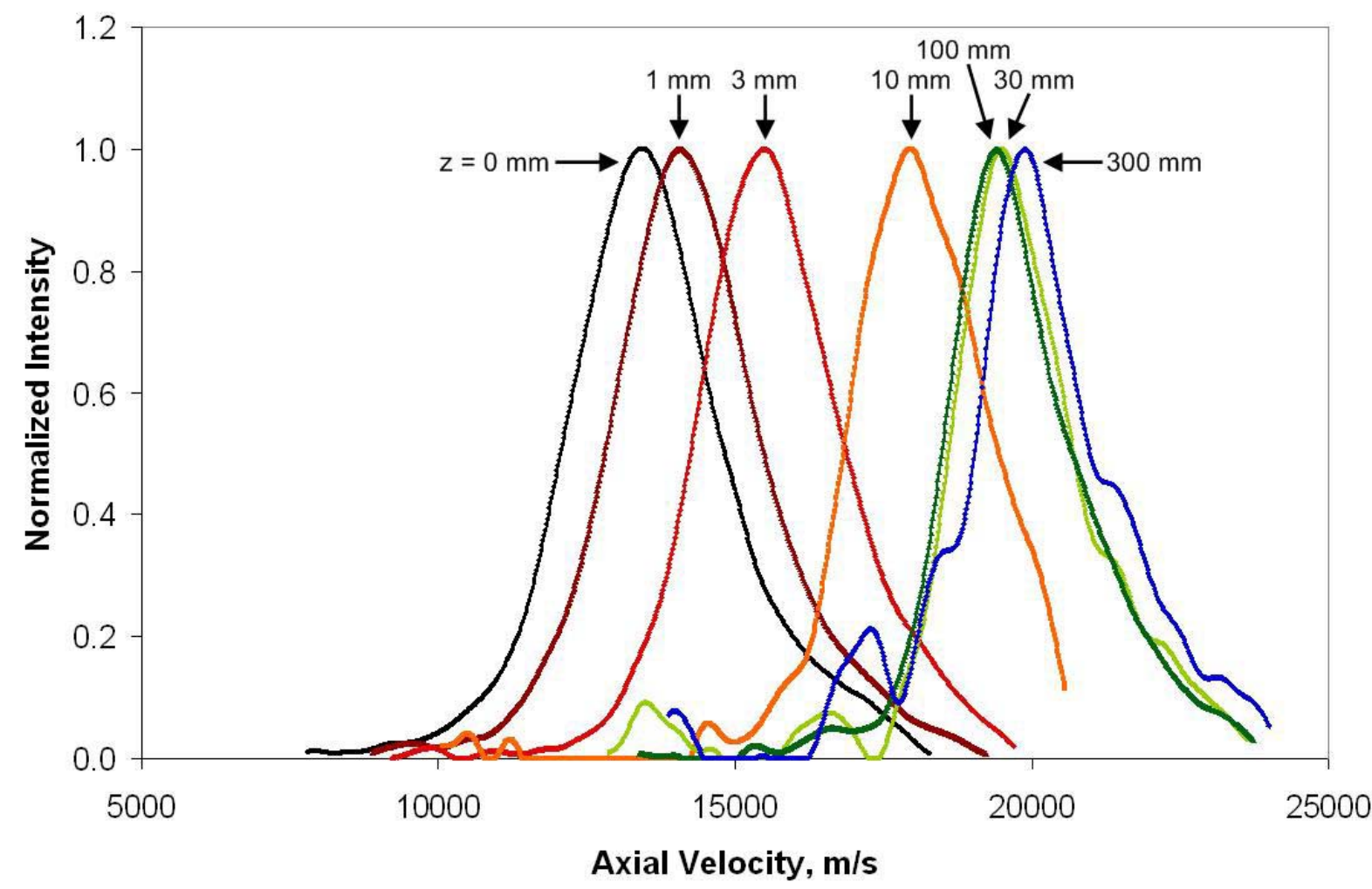

Figure 13. Velocity distribution functions along discharge channel centerline downstream of the 6-kW thruster operating at $300 \mathrm{~V}, 20 \mathrm{mg} / \mathrm{s}(\mathrm{z}=0 \mathrm{~mm}$ is the exit plane, $\mathrm{z}$ is positive downstream of the exit plane).

One possible explanation for the high-energy tail is charge-exchange collisions that turn fast doubly-charged ions into singly-charged ions. The energy for such an interaction fits well with the location of the high-energy bump on the $0-\mathrm{mm}$ scan. However, the bump seems to retract into the main VDF body as the interrogation volume moves downstream. This trend may imply that such charge-exchange collisions mostly happen very close to the thruster exit plane (and possibly in the thruster) and not appreciably in the near-field. Note from Fig. 13 that the FWHM of the VDF shrinks as the entire ion population is accelerated. This trend can be explained by kinematic compression. However, kinematic compression also tends to skew a distribution negatively. Since the VDFs shown are positiveskewed, we conclude that the highenergy tails are real and not an artifact of kinematic compression.

Figure 14 shows selected VDFs from a radial sweep across the exit plane of the thruster during a $300 \mathrm{~V}, 20 \mathrm{mg} / \mathrm{s}$ discharge. Note that while the VDF for the centerline population has a highenergy tail, the VDFs for the edge populations have low-energy tails. Due to low SNR near the walls, the corresponding two VDFs appear to be bumpy (even after the use of a Gaussian

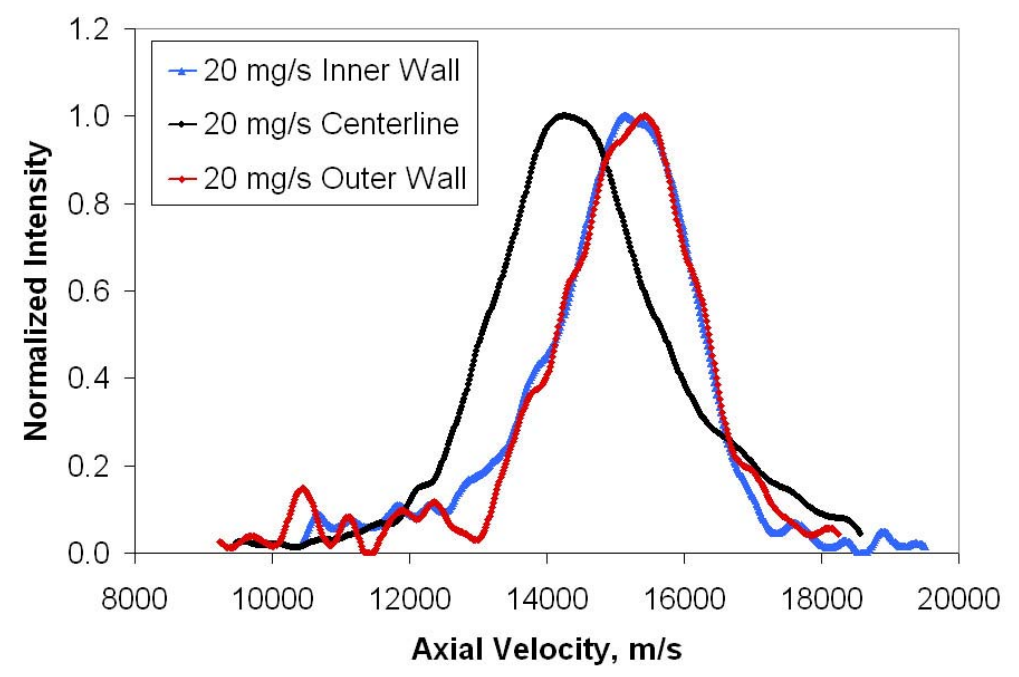

Figure 14. Velocity distribution functions at the inner wall, centerline, and outer wall of discharge channel at the exit plane with discharge at $300 \mathrm{~V}, 20 \mathrm{mg} / \mathrm{s}$ anode mass flow rate. 
filter). The VDFs for interior positions look mostly like the centerline VDF. Low-energy tails only appears when an interior scan was near the walls. A possible explanation for the low-energy tails in near-wall scans is the presence of ions that have lost momentum from colliding with the walls.

\section{Conclusion}

LIF velocimetry was performed on a 6-kW Hall thruster. A relatively Doppler-free kernel obtained by IMOG spectroscopy was used to deconvolve velocity distributions from LIF spectra. Bulk axial velocities measured along the thruster channel centerline suggested that the acceleration zone of the thruster was on the order of few tens of millimeters in length and that the loss voltage was approximately $30 \mathrm{~V}$. Radial sweeps at the exit plane revealed a slight dip in the bulk axial velocity at the center of the channel that was predicted by the magnetic field topology of the thruster. Velocity distribution functions along the centerline revealed what might be a non-trivial charge exchange population resulting from doubly-charged to singly-charged collisions. This population appeared to have been formed mostly in the thruster. Velocity distributions near the wall at the exit plane exhibited low-energy tails that might be explained by wall collisions.

\section{Acknowledgments}

The authors would like to acknowledge and thank the Air Force Office of Scientific Research (AFOSR) for funding this research. Dr. Mitat Birkan is the Project Manager for Grant FA9550-06-1-0105. The first author would also like to acknowledge the University of Michigan, Aerospace Engineering department for the Regent fellowship.

\section{References}

${ }^{1}$ Hofer, R. R., and Gallimore, A. D., "High-Specific Impulse Hall Thrusters, Part 2: Efficiency Analysis," Journal of Propulsion and Power, Vol. 22, No. 4, Jul.-Aug. 2006, pp. 732-740.

${ }^{2}$ Smith, T. B., Herman, D. A., Gallimore, A. D., and Drake, P. R., "Deconvolution of Axial Velocity Distributions from Hall Thruster LIF Spectra," 27 $7^{\text {th }}$ International Electric Propulsion Conference, IEPC-01-0019, Pasadena, CA, 15-19 Oct. 2001.

${ }^{3}$ Hargus, W. A., Jr., and Nakles, M. R., "Ion Velocity Measurements with in the Acceleration Channel of a Low Power Hall Thruster," 30 $0^{\text {th }}$ International Electric Propulsion Conference, IEPC-2007-172, Florence, Italy, 17-20 Sept. 2007.

${ }^{4}$ Cedolin, R. J., Hargus, W. A., Jr., Storm, P. V., Hanson, R. K., and Cappelli, M. A., "Laser-induced fluorescence study of a xenon Hall thruster," Applied Physics B: Lasers and Optics, Vol. 65, No. 4-5, Oct. 1997, pp. 459-469.

${ }^{5}$ Svanberg, S., Atomic and Molecular Spectroscopy, $4^{\text {th }}$ ed., Springer-Verlag, New York, 2004, Chaps. $2,9$.

${ }^{6}$ Harkness, H. W., and Heard, J. F., "The Stark Effect for Xenon," Proceedings of the Royal Society of London. Series A, Vol. 139, No. 838, 1 Feb. 1933, pp. 416-435.

${ }^{7}$ Smith, T. B., Huang, W., Ngom, B. B., and Gallimore, A. D., "Optogalvanic and laser-induced fluorescence spectroscopy of the Zeeman effect in xenon," 30 International Electric Propulsion Conference, IEPC-2007-229, Florence, Italy, 17-20 Sept. 2007.

${ }^{8}$ Ngom, B. B., Smith, T. B., Huang, W., and Gallimore, A. D., "Numerical Simulation of the Zeeman Effect from Laserinduced Fluorescence of Neutral Xenon," 30 International Electric Propulsion Conference, IEPC-2007-254, Florence, Italy, 1720 Sept. 2007.

${ }^{9}$ Hofer, R. R., Jankovsky, R. S., and Gallimore, A. D., "High-Specific Impulse Hall Thruster, Part 1: Influence of Current Density and Magnetic Field," Journal of Propulsion and Power, Vol. 22, No. 4, Jul.-Aug. 2006, pp. 721-731.

${ }^{10}$ Morosov, A. I., "Focusing of Cold Quasineutral Beams in Electromagnetic Fields," Soviet Physics-Doklady, Vol. 10, No. 8, Feb. 1966, pp. 775-777.

${ }^{11}$ Smith, T. B., Deconvolution of Ion Velocity Distributions from Laser-Induced Fluorescence Spectra of Xenon Electrostatic Thruster Plumes, Ph.D. Dissertation, University of Michigan, Ann Arbor, MI, 2003.

${ }^{12}$ Smith, T. B., Huang, W., Reid, B. M., and Gallimore, A. D., "Near-field Laser-induced Fluorescence Velocimetry of Neutral Xenon in a 6-kW Hall Thruster Plume," $30^{\text {th }}$ International Electric Propulsion Conference, IEPC-2007-252, Florence, Italy, 17-20 Sept. 2007.

${ }^{13}$ Reid, B. M., and Gallimore, A. D., "Review of Hall Thruster Neutral Flow Dynamics," $30^{\text {th }}$ International Electric Propulsion Conference, IEPC-2007-38, Florence, Italy, 17-20 Sept. 2007.

${ }^{14}$ Brown, D. L., Larson, C. W., Hass, J. M., and Gallimore, A. D., "Analytical Extraction of Plasma Properties Using a Hall Thruster Efficiency Architecture," 30 ${ }^{\text {th }}$ International Electric Propulsion Conference, IEPC-2007-188, Florence, Italy, 17-20 Sept. 2007.

${ }^{15}$ Savitzky, A, and Golay, M. J. E., "Smoothing and Differentiation of Data by Simplified Least Squares Procedures," Analytical Chemistry, Vol. 36, No. 8, Jul. 1964, pp. 1627-1639.

${ }^{16}$ Williams, G. J., Jr., Smith, T. B., Gulczinski, F. S., III, and Gallimore, A. D., "Correlating Laser-Induced Fluorescence and Molecular Beam Mass Spectrometry Ion Energy Distributions," Journal of Propulsion and Power, Vol. 18, No. 2, Mar.-Apr. 2002, pp. 489-491. 
${ }^{17}$ Reid, B. M., and Gallimore, A. D., "Plasma Potential Measurements in the Discharge Channel of a 6-kW Hall Thruster," 44 $4^{\text {th }}$ AIAA/ASME/SAE/ASEE Joint Propulsion Conference, AIAA-2008-5185, Hartford, CT, 21-23 Jul., 2008. 